\title{
Correlation of Optic Nerve Microcirculation with Papillomacular Bundle Structure in Treatment Naive Normal Tension Glaucoma
}

\author{
Wataru Kobayashi, ${ }^{1}$ Hiroshi Kunikata, ${ }^{1,2}$ Kazuko Omodaka, ${ }^{1}$ Kyousuke Togashi, ${ }^{3}$ \\ Morin Ryu, ${ }^{1}$ Masahiro Akiba, ${ }^{4}$ Gaku Takeuchi, ${ }^{4}$ Tetsuya Yuasa, ${ }^{3}$ and Toru Nakazawa ${ }^{1,2,5}$ \\ ${ }^{1}$ Department of Ophthalmology, Tohoku University Graduate School of Medicine, 1-1 Seiryo-machi, Aoba-ku, \\ Sendai 980-8574, Japan \\ ${ }^{2}$ Department of Retinal Disease Control, Ophthalmology, Tohoku University Graduate School of Medicine, 1-1 Seiryo-machi, \\ Aoba-ku, Sendai 980-8574, Japan \\ ${ }^{3}$ Department of Bio-Systems Engineering, Faculty of Engineering, Graduate School of Science and Engineering, \\ Yamagata University, 4-3-16 Jonan, Yonezawa-shi, Yamagata 992-8510, Japan \\ ${ }^{4}$ Topcon Corporation, 75-1 Hasunuma-cho, Itabashi-ku, Tokyo 174-8580, Japan \\ ${ }^{5}$ Department of Advanced Ophthalmic Medicine, Tohoku University Graduate School of Medicine, 1-1 Seiryo-machi, \\ Aoba-ku, Sendai 980-8574, Japan \\ Correspondence should be addressed to Toru Nakazawa; ntoru@oph.med.tohoku.ac.jp
}

Received 22 August 2014; Accepted 21 November 2014; Published 9 December 2014

Academic Editor: Rachel W. Kuchtey

Copyright ( 2014 Wataru Kobayashi et al. This is an open access article distributed under the Creative Commons Attribution License, which permits unrestricted use, distribution, and reproduction in any medium, provided the original work is properly cited.

Purpose. To assess the association between optic nerve head (ONH) microcirculation, central papillomacular bundle (CPB) structure, and visual function in eyes with treatment naive normal tension glaucoma (NTG). Methods. This study included 40 eyes of 40 patients with NTG and 20 eyes of 20 normal patients. We used laser speckle flowgraphy (LSFG) to measure mean blur rate (MBR) in all eyes and calculated the ratio of MBR in the horizontal quadrants of tissue area ONH (temporal/nasal ratio of MBR in the tissue area: T/N MT). Clinical findings also included retinal nerve fiber layer thickness (RNFLT) and ganglion cell complex thickness (GCCT) in the CPB and macular areas, best-corrected visual acuity (BCVA), mean deviation (MD), and refractive error. Results. T/N MT was correlated with both BCVA and MD. The OCT parameters most highly correlated with T/N MT were macular RNFLT and mid-CPB RNFLT. Furthermore, T/N MT, mid-CPB RNFLT, and macular RNFLT were higher in NTG than in normal eyes. A discrimination analysis revealed that T/N MT and refractive error were independent factors indicating NTG. Conclusions. Our results suggest that T/N MT is a candidate biomarker of NTG. Furthermore, T/N MT reflects visual function, including acuity and sensitivity, and $\mathrm{CPB}$ structure.

\section{Introduction}

Glaucoma affects over 70 million people and is the second most common cause of blindness worldwide $[1,2]$. Normal tension glaucoma (NTG), despite being the most common type of glaucoma in Asia, is less well understood than primary open angle glaucoma (POAG) [3-5]. Previous reports have shown that there is a general relationship between optic nerve microcirculation and glaucoma [6-9]. This relationship, in particular the effect of decreased microcirculation in the optic nerve head $(\mathrm{ONH})$ in glaucoma, is one of the most promising potential sources of new insights into the pathogenesis of NTG, along with such factors as oxidative stress and axonal flow impairment. Quick, easy, and noninvasive ways to measure $\mathrm{ONH}$ microcirculation would therefore be very helpful for the investigation of the role of reduced $\mathrm{ONH}$ blood flow in glaucoma.

Laser speckle flowgraphy (LSFG) is a promising method of measuring $\mathrm{ONH}$ microcirculation that relies on the laser speckle phenomenon. Recent innovations in LSFG have 
allowed the in vivo quantification of microcirculation in the $\mathrm{ONH}$, choroid, and retinal vessels separately $[10,11]$. The main measurement parameter of LSFG is mean blur rate (MBR) [12, 13], a quantitative index of retinal blood cell (RBC) velocity. MBR is currently used mainly to monitor changes over time in $\mathrm{ONH}$ or choroidal microcirculation in the same eye at the same site [11], because the laser speckle signal can be influenced not only by RBC velocity, but also by laser reflectance and absorption in the retinal tissue [10]. Thus, previous interindividual comparisons of LSFG data have relied on analyses of $\mathrm{MBR}$ waveforms or MBR ratios rather than direct analysis of quantitative MBR values [14, $15]$.

Changes in the retinal structure, including macular and circumpapillary retinal nerve fiber layer thickness (mRNFLT and cpRNFLT, resp.), have also been reported to be closely associated with NTG [16-18]. Previously, we demonstrated that temporal cpRNFLT is significantly correlated to visual acuity in patients with glaucoma [19]. This prompted the current investigation, in which we compared the ratio of MBR data for the temporal and nasal optic nerve, measured with LSFG, and the thickness of the RNFL and GCC in the macula and the central papillomacular bundle (CPB), using a newly developed OCT analysis program. We also investigated differences in these values in NTG patients and normal subjects. Additionally, our analysis included clinical findings such as visual acuity and standard automated perimetry (SAP) measurements of mean deviation (MD). The purpose of this study was thus to evaluate the relationship between optic nerve microcirculation and retinal structure/function in eyes with treatment naive NTG.

\section{Subjects and Methods}

2.1. Inclusion Criteria. This retrospective, cross-sectional study comprised 40 eyes of 40 Japanese adult patients with NTG. Data from 20 eyes of 20 normal subjects ( $>40$ years old) were used for comparison. All the NTG patients exhibited glaucomatous optic neuropathy. The inclusion criteria were (1) treatment naive NTG, (2) age > 40 years old, (3) a spherical equivalent refractive error of $>-7.00$ diopters, and (4) a glaucomatous visual field meeting the AndersonPatella classification. The exclusion criteria were (1) decimal visual acuity $<0.1,(2)$ cataracts with severity greater than grade 2 of the Emery-Little classification, and (3) the presence of macular diseases such as macular edema, macular degeneration, or epiretinal membrane. The baseline clinical parameters recorded for each patient were age, sex, and refractive error. The baseline best-corrected visual acuity (BCVA) was measured with a standard Japanese decimal visual acuity chart and converted to logarithm of the minimum angle of resolution (logMAR) for statistical analysis. IOP was measured with Goldmann applanation tonometry during the initial diagnosis of NTG, before any glaucoma medications were used by the patient. The study adhered to the tenets of the Declaration of Helsinki, and the protocols were approved by the Clinical Research Ethics Committee of Tohoku University Graduate School of Medicine.
2.2. Visual Field Analysis. MD was measured with the 30-2 program of the Humphrey field analyzer (HFA; Carl Zeiss Meditec, Dublin, CA, USA), using the Swedish interactive threshold algorithm (SITA) standard strategy. HFA examinations were performed within three months of the OCT measurements. Only reliable MD values were used, excluding examinations with $<20 \%$ fixation errors and $<33 \%$ falsepositives or false-negatives.

2.3. Laser Speckle Flowgraphy. ONH microcirculation was evaluated by measuring MBR in the optic disc with LSFGNAVI (Softcare Ltd., Fukuoka, Japan). Ophthalmic examinations including slit-lamp biomicroscopy and gonioscopy were performed, and patients with narrow angles were excluded. LSFG measurements were carried out after dilation of the pupil with $0.4 \%$ tropicamide (Midrin-M, Santen Pharmaceutical Co. Ltd., Osaka, Japan). Before the LSFG examination, the patients rested on a chair with their eyes closed for $10 \mathrm{~min}$ utes in a dark room and measured their blood pressure. All examinations were performed by experienced investigators. Edge detection of the optic disc in the MBR image was performed manually and the disc edge was saved in software. The vessels were then segmented in the supplied software (LSFG Analyzer, v 3.0.47.0) with an automated defining threshold, and the values of mean MBR (MA), MBR in the vessel area $(\mathrm{MV})$, and MBR in tissue area (MT) were determined. These values were determined separately for each quadrant of the ONH: superior (S), inferior (I), temporal (T), and nasal area $(\mathrm{N})$, as well as overall. Triplicate measurements were made of each subject, separated by several minutes, using the saved data for the optic disc edge.

2.4. OCT Scanning of the Disc and Macular Areas. CpRNFLT, mRNFLT, and GCCT were determined with 3D OCT-2000 software (version 8.00; Topcon Inc.). After obtaining circle scans and macular cube scans (in a $7 \times 7 \mathrm{~mm}$ area corresponding to 10-degree square area of the macula) centered on the fovea, the software automatically calculated the thickness of each layer.

2.5. OCT Scanning of the Central Papillomacular Bundle. The $\mathrm{CPB}$ was defined in this study as a $1.5 \times 9.0 \mathrm{~mm}$ rectangular area centered between the optic nerve disc and the macula, aligned perpendicularly to the nerve fibers. At either end of the scan area, a $1.5 \times 1.2 \mathrm{~mm}$ area in which the retinal layers could not be reliably segmented was discarded. The remaining $1.5 \times 6.6 \mathrm{~mm}$ area was divided lengthwise into three $1.5 \times$ $2.2 \mathrm{~mm}$ sections, representing the upper, middle, and lower $\mathrm{CPB}$. Analysis of the CPB used 3D OCT images, obtained with the 3D OCT-2000 (Topcon Corporation, Tokyo, Japan) device. Each image was constructed from 64 B-scan images (pixel dimensions: $512 \times 885$, grayscale levels: 256 ) with depth and lateral resolutions of $6 \mu \mathrm{m}$ and $20 \mu \mathrm{m}$, respectively. Layer segmentation was performed with newly developed software (Topcon). The RNFLT and GCCT of the CPB were measured with automatic analysis software developed by the Graduate School of Science and Engineering, Yamagata University. This software was equipped with a registration system (using a 
fast registration algorithm for the 3D OCT images based on en-face projection images) to ensure the reliability and reproducibility of the clinical data.

2.6. Statistical Analysis. Spearman's correlation analysis was used to determine the correlation between the data from the structural examinations, that is, RNFLT and GCCT, and the data from the functional tests, that is, MD and BCVA $(\log M A R)$, as well as the ratio of tissue area MBR in the horizontal quadrants, that is, the temporal and nasal tissue area MBR (T/N MT). The Mann-Whitney $U$ test was used to determine differences between the NTG patients and normal subjects. A discrimination analysis was used to determine the predictive power of the measurement parameters for NTG. The analysis used JMP Pro version 9.0.2 software for Windows (SAS Institute, Japan). A $P$ value $<0.05$ was considered to be statistically significant.

\section{Results}

Table 1 shows demographic information and biometric parameters for the NTG patients and normal subjects. Table 2 shows the correlations of these parameters with T MT, I MT, N MT, S MT, and T/N MT. T MT was most closely correlated with all but one of the OCT parameters. T/N MT was also closely correlated with all parameters. The parameters most strongly correlated with T/N MT were mid-CPB RNFLT, mRNFLT, mid-CPB GCCT, and macular GCCT $(r=0.62$, $r=0.66, r=0.57$, and $r=0.56$, resp., all $P<0.01$ ). Figure 1 shows comparisons of T/N MT and retinal thickness (in the mid-CPB and macular areas) in NTG patients and normal subjects. We found significant differences between the NTG patients and normal subjects in T/N MT and mid-CPB and macular retinal thickness (all $P<0.01$ ). Figure 2 shows the correlation of T/N MT and retinal thickness (in the mid$\mathrm{CPB}$ and macular areas) and MD and BCVA (logMAR) (all $P<0.01$ ).

A discrimination analysis including 20 NTG patients and 20 age-matched normal subjects revealed that T/N MT and refractive error were independent factors able to distinguish NTG patients from normal subjects $(P<0.05$, Table 3$)$. The hit ratio of correctly classified cases was $80.0 \%$.

\section{Discussion}

In this study, we examined the relationship between novel analytic parameters of temporal optic nerve microcirculation and papillomacular bundle thickness in NTG patients. Among these parameters, we found that T/N MT, the ratio of optic nerve microcirculation in the temporal quadrant, was strongly correlated with RNFLT in the mid-CPB and macular areas and with GCCT in the mid-CPB and macular areas. We also found correlations between T/N MT and total mid-CPB and macular thickness as well as correlations between $\mathrm{T} / \mathrm{N}$ MT and visual function, including visual acuity and MD.

It is well known that, in glaucoma, the eye undergoes loss of the retinal GCL and of the axons that comprise the RNFL [20]. The pathogenesis of these phenomena remains unclear, but changes in ocular blood flow, especially decreases in $\mathrm{ONH}$ microcirculation, have recently been reported to play an important role, particularly in the occurrence and progression of NTG $[21,22]$. This new understanding of the role of ocular blood flow may help explain why lowering IOP treatment, despite being an effective, gold-standard treatment for POAG, has limitations when used for NTG [23]. Epidemiological studies including the Singapore Malays Eyes Study [24] and the Beijing Eye Study [25] have shown that the prevalence of glaucoma increases with retinal arteriole or vessel narrowing, and the Blue Mountain Eye Study showed that glaucoma was 2.7 times more likely in eyes with retinal arteriole narrowing [26]. A review of recent research on glaucoma revealed a number of reports on the role of dysfunctional ocular blood flow in the prevalence and progression of glaucoma [27-29], reinforcing our findings. Previous findings in which the highest correlation between visual acuity and RNFLT was in the temporal sector prompted our study's focus on temporal ONH microcirculation [19]. As it is well known that visual acuity decreases only in the end stages of glaucoma, it has been thought that the macula in eyes with glaucoma only rarely becomes disordered [30]. In fact, recent reports have shown that damage to the macula can occur even in the early stages of glaucoma [31,32]. Our study may help explain these previous findings, as our results suggest that decreases in temporal optic nerve blood flow may affect the structure of the $\mathrm{CPB}$ and macula, leading to decreases in macular function, including visual acuity.

Laser speckle flowgraphy (LSFG) is a new and increasingly commonly used technology that can quantify microcirculation in the optic disc, choroid, and retinal vessels separately. It is noninvasive, accurate, and flexible and is therefore suitable for studying many aspects of ocular circulation [11]. $\mathrm{MBR}$, the main measurement parameter of LSFG, is a relative measurement index of the velocity of erythrocytes [12, 13]. $\mathrm{ONH}$ filling defect area, measured with fluorescein angiography (FA), is also used in studies of ocular blood flow and is correlated to glaucomatous damage [33-37]. It is difficult to include FA as a part of clinical care for glaucoma, however, as quantitative measurements of changes in ONH blood flow cannot be obtained with sufficient reliability. Other methods to quantify blood flow have also been reported, such as scanning laser ophthalmoscopy-FA, which can measure decreases in fluorescein velocity in eyes with glaucoma [8], singlepoint laser Doppler flowmetry, which can identify decreased ONH blood flow in glaucoma [6], and scanning laser Doppler flowmetry, which has been used to show that retinal blood flow in both the neuroretinal rim and peripapillary area is decreased in glaucoma [7]. Scanning laser Doppler flowmetry has also been used to show that perfusion in the $\mathrm{ONH}$ can decrease before the manifestation of visual field defects [38]. In contrast with these techniques, LSFG measurements of MBR can be obtained easily and quickly (within a few seconds) and are highly reproducible in both normal and glaucoma subjects [39]. In our study, we investigated the role of ocular microcirculation in glaucoma by determining the ratio of $\mathrm{MBR}$ in the temporal and nasal quadrants of the $\mathrm{ONH}$ tissue area. We found that, among the quadrants of the $\mathrm{ONH}$, temporal MBR was most closely correlated with a number 
TABLE 1: Characteristics of patients.

\begin{tabular}{|c|c|c|c|}
\hline Characteristics & $\begin{array}{c}\text { NTG } \\
\text { Number, mean } \pm \text { SD }\end{array}$ & $\begin{array}{c}\text { Normal people } \\
\text { Number, mean } \pm \text { SD }\end{array}$ & $P$ value \\
\hline Number & 40 & 20 & - \\
\hline \multicolumn{4}{|l|}{ Gender } \\
\hline Male & 19 (19 eyes) & 10 (10 eyes) & \multirow{2}{*}{$0.81^{*}$} \\
\hline Female & 21 (21 eyes) & 10 (10 eyes) & \\
\hline \multicolumn{4}{|l|}{ Laterality } \\
\hline Right & 21 & 9 & \multirow{2}{*}{$0.81^{*}$} \\
\hline Left & 19 & 11 & \\
\hline Age (years) & $63.3 \pm 9.6$ & $59.7 \pm 9.9$ & $0.16^{* *}$ \\
\hline BCVA (log MAR) & $0.10 \pm 0.2$ & $-0.10 \pm 0.08$ & $<0.01^{* *}$ \\
\hline IOP (mmHg) & $14.6 \pm 3.2$ & $16.2 \pm 3.3$ & $0.18^{* *}$ \\
\hline Refractive error (diopter) & $-2.94 \pm 2.3$ & $-2.04 \pm 2.2$ & $0.17^{* *}$ \\
\hline HFA $30-2$ mean deviation (MD; dB) & $-11.9 \pm 8.4$ & $0.17 \pm 1.3$ & $<0.01^{* *}$ \\
\hline \multicolumn{4}{|l|}{ Macular area } \\
\hline RNFLT $(\mu \mathrm{m})$ & $19.1 \pm 9.1$ & $34.5 \pm 4.0$ & $<0.01^{* *}$ \\
\hline $\operatorname{GCCT}(\mu \mathrm{m})$ & $75.3 \pm 14.7$ & $101.6 \pm 7.0$ & $<0.01^{* *}$ \\
\hline \multicolumn{4}{|l|}{ Central papillomacular bundle } \\
\hline \multicolumn{4}{|l|}{ RNFLT $(\mu \mathrm{m})$} \\
\hline Upper & $36.4 \pm 21.2$ & $66.9 \pm 15.6$ & $<0.01^{* *}$ \\
\hline Middle & $30.3 \pm 13.9$ & $42.2 \pm 7.7$ & $<0.01^{* *}$ \\
\hline Lower & $29.1 \pm 21.0$ & $70.9 \pm 18.1$ & $<0.01^{* *}$ \\
\hline \multicolumn{4}{|l|}{$\operatorname{GCCT}(\mu \mathrm{m})$} \\
\hline Upper & $81.5 \pm 25.1$ & $117.2 \pm 14.2$ & $<0.01^{* *}$ \\
\hline Middle & $90.7 \pm 24.9$ & $114.5 \pm 9.5$ & $<0.01^{* *}$ \\
\hline Lower & $75.3 \pm 21.4$ & $120.7 \pm 17.7$ & $<0.01^{* *}$ \\
\hline \multicolumn{4}{|l|}{ Circumpapillary RNFLT $(\mu \mathrm{m})$} \\
\hline Total & $81.2 \pm 12.2$ & $107.3 \pm 10.6$ & $<0.01^{* *}$ \\
\hline Temporal & $71.1 \pm 17.5$ & $84.9 \pm 11.5$ & $<0.01^{* *}$ \\
\hline Superior & $98.3 \pm 19.2$ & $126.6 \pm 16.3$ & $<0.01^{* *}$ \\
\hline Nasal & $75.1 \pm 16.3$ & $86.1 \pm 15.9$ & $<0.01^{* *}$ \\
\hline Inferior & $80.4 \pm 20.6$ & $131.5 \pm 17.0$ & $<0.01^{* *}$ \\
\hline \multicolumn{4}{|l|}{ LSFG MBR (AU) } \\
\hline \multicolumn{4}{|l|}{ MA } \\
\hline Temporal & $8.6 \pm 2.6$ & $11.8 \pm 2.1$ & - \\
\hline Superior & $20.4 \pm 6.1$ & $28.3 \pm 5.6$ & - \\
\hline Nasal & $21.5 \pm 6.3$ & $29.9 \pm 6.4$ & - \\
\hline Inferior & $17.5 \pm 5.0$ & $26.7 \pm 5.3$ & - \\
\hline \multicolumn{4}{|l|}{ MT } \\
\hline Temporal & $7.8 \pm 2.0$ & $10.1 \pm 1.6$ & - \\
\hline Superior & $10.8 \pm 2.7$ & $12.6 \pm 2.0$ & - \\
\hline Nasal & $11.9 \pm 2.5$ & $13.5 \pm 1.6$ & - \\
\hline Inferior & $10.0 \pm 2.4$ & $12.9 \pm 1.8$ & - \\
\hline \multicolumn{4}{|l|}{ MV } \\
\hline Temporal & $27.2 \pm 10.1$ & $36.9 \pm 12.7$ & - \\
\hline Superior & $37.9 \pm 9.0$ & $48.0 \pm 5.2$ & - \\
\hline Nasal & $36.3 \pm 8.9$ & $48.4 \pm 7.6$ & - \\
\hline Inferior & $35.1 \pm 7.7$ & $44.7 \pm 5.1$ & - \\
\hline Temporal/nasal MT & $0.66 \pm 0.1$ & $0.75 \pm 0.1$ & $<0.01^{* *}$ \\
\hline Systolic blood pressure (SBP) (mmHg) & $127.1 \pm 20.4$ & $133.7 \pm 21.7$ & $0.26^{* *}$ \\
\hline Diastolic blood pressure (DBP) (mmHg) & $77.8 \pm 14.5$ & $82.9 \pm 14.1$ & $0.07^{* *}$ \\
\hline Heart rate (HR) (bpm) & $73.2 \pm 11.1$ & $76.9 \pm 9.9$ & $0.16^{* *}$ \\
\hline
\end{tabular}




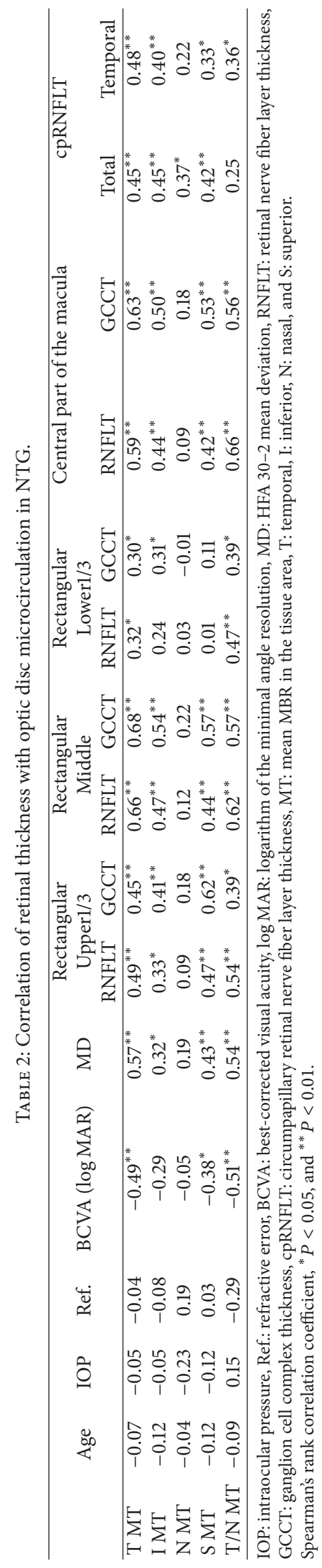



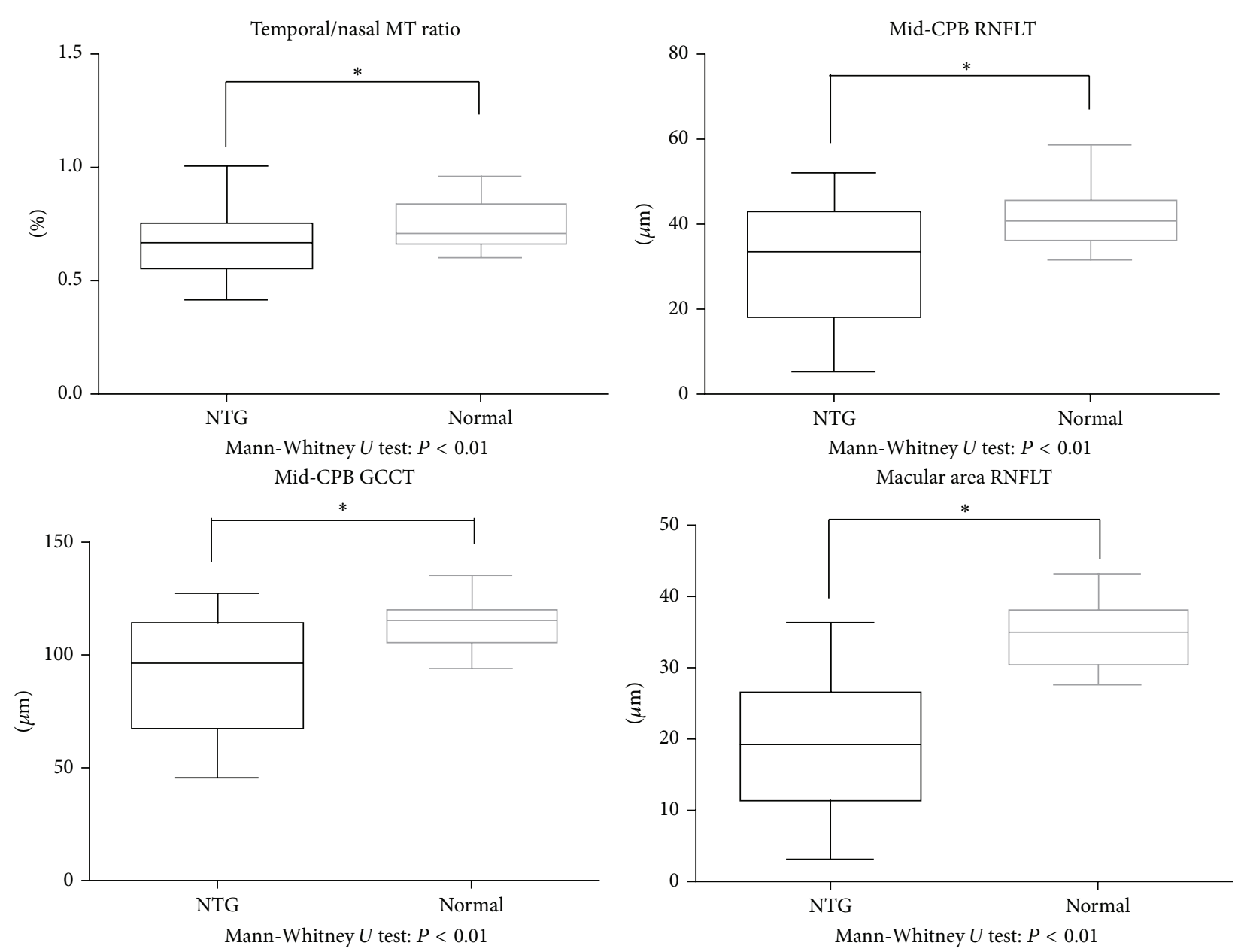

Macular area GCCT

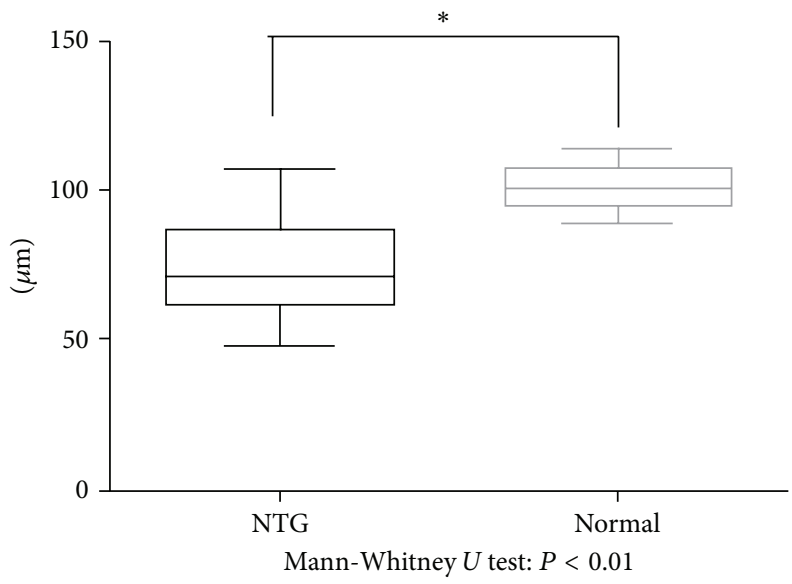

FIGURE 1: Comparison of T/N MT, RNFLT, and GCCT in NTG patients and normal subjects. T/N MT, mid-CPB RNFLT, mid-CPB GCCT, macular RNFLT, and macular GCCT were decreased in NTG patients compared to normal subjects (all $P<0.01$ ).

of parameters of retinal thickness, while nasal MBR was the least. We thus calculated the ratio between MBR in these two quadrants in order to obtain a value that was comparable between eyes. The correlation between temporal MBR and other measurement parameters reflects the close relationship between temporal ONH microcirculation and central visual function, while the lack of correlation of nasal MBR reflects the common preservation of nasal ONH microcirculation in glaucoma patients, despite the loss of visual function $[14,19]$.

Currently, the diagnosis of glaucoma is mainly made based on the presence of pronounced optic disc cupping, decreased cpRNFLT, and progressive visual field loss. Recently, spectral domain optical coherence tomography (SD-OCT) technology has been introduced, which enables 

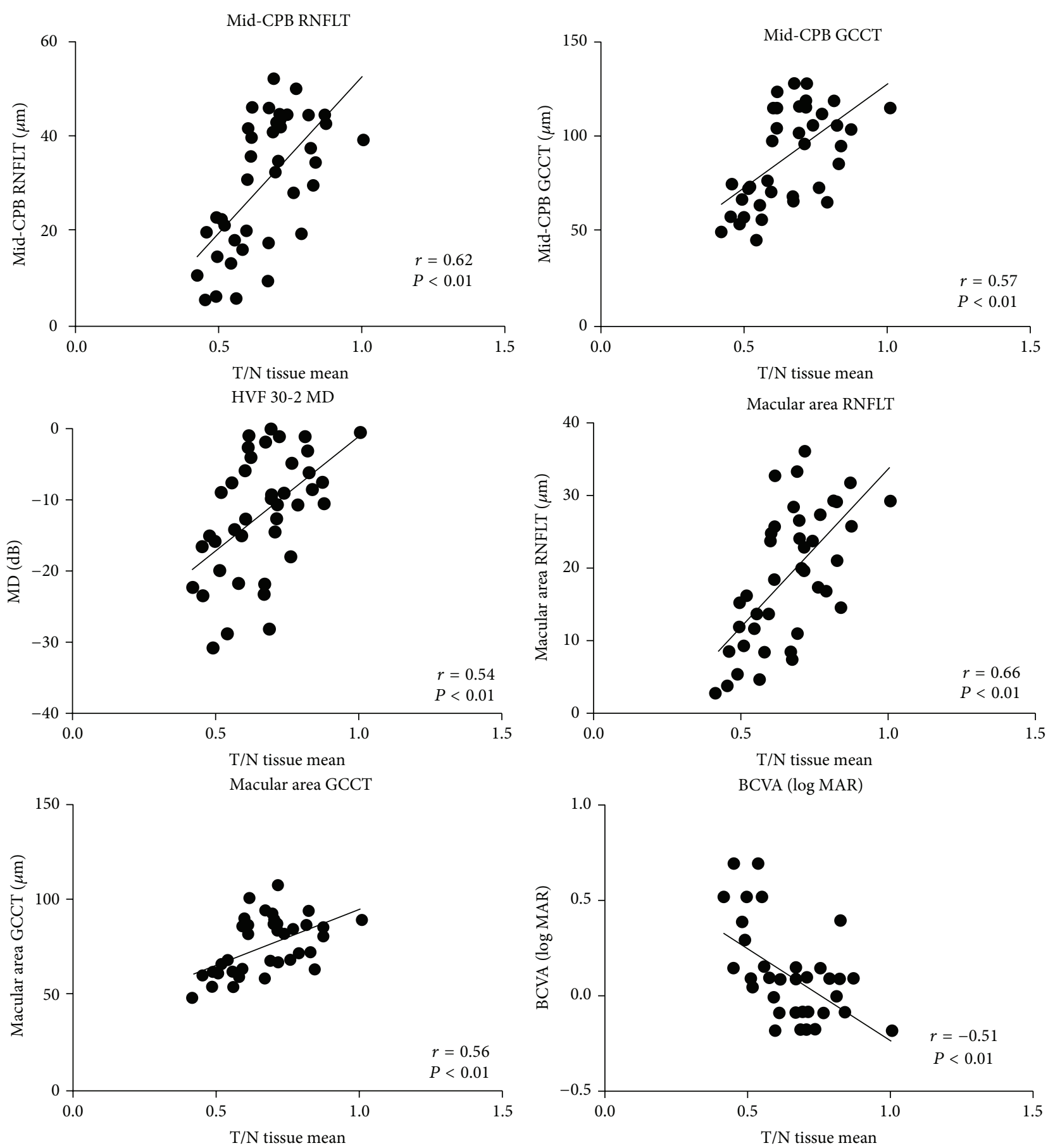

Mid-CPB: middle central papillomacular bundle RNFLT: retinal nerve fiber layer thickness GCCT: ganglion cell complex thickness Spearman's rank correlation coefficient

Mid-CPB: middle central papillomacular bundle RNFLT: retinal nerve fiber layer thickness GCCT: ganglion cell complex thickness Spearman's rank correlation coefficient

Figure 2: Correlation of T/N MT with MD, BCVA, RNFLT, and GCCT. In NTG patients, T/N MT was correlated with mid-CPB RNFLT, mid-CPB GCCT, macular RNFLT, macular GCCT, MD, and BCVA (all $P<0.01$ ).

the visualization of each retinal layer in the macular area, including the GCC, with programmed segmentation algorithms [40]. In glaucoma patients, the macular area is impaired because of changes in the RNFL and GCC of the inner retina [41]. Several studies have reported that macular
GCCT and cpRNFLT have a very similar diagnostic potential for glaucoma $[16,17,41]$. The current study both confirmed the value of conventional measurements of macular thickness and found that a new measurement program for $\mathrm{CPB}$ thickness may also be helpful, as it was closely correlated 
TABLE 3: Results of discrimination analysis for factors independently contributing to NTG.

(a)

\begin{tabular}{lccc}
\hline Variable & Wilks's lambda & $F$-number & $P$ value \\
\hline Age & 0.9560 & 1.6104 & 0.32 \\
IOP & 0.8967 & 4.0329 & 0.06 \\
Ref. & 0.8383 & 6.7496 & $<0.05$ \\
T/N MT & 0.6478 & 19.0278 & $<0.01$ \\
\hline
\end{tabular}

(b)

\begin{tabular}{lccc}
\hline Judgment result & Normal & $\begin{array}{c}\text { Predicted value } \\
\text { NTG }\end{array}$ & Hit ratio \\
\hline Observed value & & & \\
$\quad$ Normal & 16 & 4 & $80.0 \%$ \\
NTG & 4 & 16 & $80.0 \%$ \\
\hline \multicolumn{2}{r}{} & Total & $80.0 \%$ \\
\hline
\end{tabular}

with temporal $\mathrm{ONH}$ circulation. This new measurement parameter could identify NTG and was associated with visual acuity and sensitivity.

This study had a number of limitations. It included only a small number of eyes with glaucoma (approximately 40) and was retrospective. The study methodology introduced the potential for bias, as myopia in the patients could have affected our results for the correlation between structure and function. We therefore excluded NTG patients with high myopia (less than -7 diopters) and there was no statistical difference on refractive error between NTG and normal subjects. Nevertheless, refractive error was present in our results as a weak independent factor differentiating NTG. Furthermore, although there were significant differences in the T/N MT ratio between normal and NTG eyes, the data for the two groups overlapped a fair amount. Nevertheless, when the analysis also includes simple clinical parameters such as age, IOP, and refractive error, its hit rate for detecting NTG is $80 \%$, sufficient for it to be considered a good clinical indicator.

In this study, we examined the relationship between structure/function and microcirculation in eyes with NTG. We found that the ratio of temporal $\mathrm{ONH}$ blood flow was strongly correlated with mid-CPB and macular thickness as well as BCVA and MD, and that measurement of temporal $\mathrm{ONH}$ microcirculation may be an excellent way to determine whether thinning of the RNFL and GCC in glaucoma patients is due to NTG.

\section{Conflict of Interests}

The authors report no conflict of interests.

\section{Acknowledgment}

This study was supported in part by research grants from the Japan Science and Technology Agency (JST) Center for Revitalization Promotion.

\section{References}

[1] H. A. Quigley, "Number of people with glaucoma worldwide," British Journal of Ophthalmology, vol. 80, no. 5, pp. 389-393, 1996.

[2] S. Resnikoff, D. Pascolini, D. Etya'ale et al., "Global data on visual impairment in the year 2002," Bulletin of the World Health Organization, vol. 82, no. 11, pp. 844-851, 2004.

[3] A. Iwase, Y. Suzuki, M. Araie et al., "The prevalence of primary open-angle glaucoma in Japanese: the Tajimi Study," Ophthalmology, vol. 111, no. 9, pp. 1641-1648, 2004.

[4] R. K. Na, S. L. Eun, J. S. Gong et al., "Comparing the ganglion cell complex and retinal nerve fibre layer measurements by Fourier domain OCT to detect glaucoma in high myopia," British Journal of Ophthalmology, vol. 95, no. 8, pp. 1115-1121, 2011.

[5] Y. B. Liang, D. S. Friedman, Q. Zhou et al., "Prevalence of primary open angle glaucoma in a rural adult chinese population: the Handan eye study," Investigative Ophthalmology \& Visual Science, vol. 52, no. 11, pp. 8250-8257, 2011.

[6] P. Hamard, H. Hamard, J. Dufaux, and S. Quesnot, "Optic nerve head blood flow using a laser Doppler velocimeter and haemorheology in primary open angle glaucoma and normal pressure glaucoma," British Journal of Ophthalmology, vol. 78, no. 6, pp. 449-453, 1994.

[7] G. Michelson, "Perfusion of the juxtapapillary retina and the neuroretinal rim area in primary open angle glaucoma," Journal of Glaucoma, vol. 5, no. 2, pp. 91-98, 1996.

[8] S. Wolf, O. Arend, W. E. Sponsel, K. Schulte, L. B. Cantor, and M. Reim, "Retinal hemodynamics using scanning laser ophthalmoscopy and hemorheology in chronic open-angle glaucoma," Ophthalmology, vol. 100, no. 10, pp. 1561-1566, 1993.

[9] A. Harris, R. C. Sergott, G. L. Spaeth, J. L. Katz, J. A. Shoemaker, and B. J. Martin, "Color Doppler analysis of ocular vessel blood velocity in normal-tension glaucoma," American Journal of Ophthalmology, vol. 118, no. 5, pp. 642-649, 1994.

[10] M. Nagahara, Y. Tamaki, A. Tomidokoro, and M. Araie, "In vivo measurement of blood velocity in human major retinal vessels using the laser speckle method," Investigative Ophthalmology and Visual Science, vol. 52, no. 1, pp. 87-92, 2011.

[11] T. Sugiyama, M. Araie, C. E. Riva, L. Schmetterer, and S. Orgul, "Use of laser speckle flowgraphy in ocular blood flow research," Acta Ophthalmologica, vol. 88, no. 7, pp. 723-729, 2010.

[12] G. Watanabe, H. Fujii, and S. Kishi, "Imaging of choroidal hemodynamics in eyes with polypoidal choroidal vasculopathy using laser speckle phenomenon," Japanese Journal of Ophthalmology, vol. 52, no. 3, pp. 175-181, 2008.

[13] N. Konishi, Y. Tokimoto, K. Kohra, and H. Fujii, "New laser speckle flowgraphy system using CCD camera," Optical Review, vol. 9, no. 4, pp. 163-169, 2002.

[14] N. Aizawa, H. Kunikata, K. Omodaka, and T. Nakazawa, “Optic disc microcirculation in superior segmental optic hypoplasia assessed with laser speckle flowgraphy," Clinical and Experimental Ophthalmology, vol. 42, no. 7, pp. 702-704, 2014.

[15] Y. Shiga, K. Omodaka, H. Kunikata et al., "Waveform analysis of ocular blood flow and the early detection of normal tension glaucoma," Investigative Ophthalmology \& Visual Science, vol. 54, no. 12, pp. 7699-7706, 2013.

[16] M. Seong, K. R. Sung, E. H. Choi et al., "Macular and peripapillary retinal nerve fiber layer measurements by spectral domain optical coherence tomography in normal-tension glaucoma," 
Investigative Ophthalmology and Visual Science, vol. 51, no. 3, pp. 1446-1452, 2010.

[17] N. R. Kim, E. S. Lee, G. J. Seong, J. H. Kim, H. G. An, and C. Y. Kim, "Structure-function relationship and diagnostic value of macular ganglion cell complex measurement using fourier-domain OCT in glaucoma," Investigative Ophthalmology \& Visual Science, vol. 51, no. 9, pp. 4646-4651, 2010.

[18] K. Takayama, M. Hangai, M. Durbin et al., "A novel method to detect local ganglion cell loss in early glaucoma using spectral-domain optical coherence tomography," Investigative Ophthalmology \& Visual Science, vol. 53, no. 11, pp. 6904-6913, 2012.

[19] K. Omodaka, T. Nakazawa, Y. Yokoyama, H. Doi, N. Fuse, and K. Nishida, "Correlation between peripapillary macular fiber layer thickness and visual acuity in patients with open-angle glaucoma," Clinical Ophthalmology, vol. 4, no. 1, pp. 629-635, 2010.

[20] H. A. Quigley, G. R. Dunkelberger, and W. R. Green, "Retinal ganglion cell atrophy correlated with automated perimetry in human eyes with glaucoma," The American Journal of Ophthalmology, vol. 107, no. 5, pp. 453-464, 1989.

[21] J. Flammer, S. Orgül, V. P. Costa et al., "The impact of ocular blood flow in glaucoma," Progress in Retinal and Eye Research, vol. 21, no. 4, pp. 359-393, 2002.

[22] T. Nagaoka, E. Sato, A. Takahashi, H. Yokota, K. Sogawa, and A. Yoshida, "Impaired retinal circulation in patients with type 2 diabetes mellitus: retinal laser doppler velocimetry study," Investigative Ophthalmology \& Visual Science, vol. 51, no. 12, pp. 6729-6734, 2010.

[23] Collaborative Normal-Tension Glaucoma Study Group, "Comparison of glaucomatous progression between untreated patients with normal-tension glaucoma and patients with therapeutically reduced intraocular pressures," American Journal of Ophthalmology, vol. 126, no. 4, pp. 487-497, 1998.

[24] N. Amerasinghe, T. Aung, N. Cheung et al., "Evidence of retinal vascular narrowing in glaucomatous eyes in an Asian population," Investigative Ophthalmology \& Visual Science, vol. 49, no. 12, pp. 5397-5402, 2008.

[25] S. Wang, L. Xu, Y. Wang, and J. B. Jonas, "Retinal vessel diameter in normal and glaucomatous eyes: the Beijing eye study," Clinical and Experimental Ophthalmology, vol. 35, no. 9, pp. 800-807, 2007.

[26] P. Mitchell, H. Leung, J. J. Wang et al., "Retinal vessel diameter and open-angle glaucoma: the Blue Mountains Eye Study," Ophthalmology, vol. 112, no. 2, pp. 245-250, 2005.

[27] J. Caprioli and A. L. Coleman, "Blood pressure, perfusion pressure, and glaucoma," American Journal of Ophthalmology, vol. 149, no. 5, pp. 704-712, 2010.

[28] M. C. Leske, "Ocular perfusion pressure and glaucoma: clinical trial and epidemiologic findings," Current Opinion in Ophthalmology, vol. 20, no. 2, pp. 73-78, 2009.

[29] A. Werne, A. Harris, D. Moore, I. BenZion, and B. Siesky, "The circadian variations in systemic blood pressure, ocular perfusion pressure, and ocular blood flow: risk factors for glaucoma?" Survey of Ophthalmology, vol. 53, no. 6, pp. 559-567, 2008.

[30] W. M. Hart Jr. and B. Becker, "The onset and evolution of glaucomatous visual field defects," Ophthalmology, vol. 89, no. 3, pp. 268-279, 1982.

[31] E. Chihara and H. Tanihara, "Parameters associated with papillomacular bundle defects in glaucoma," Graefe's Archive for
Clinical and Experimental Ophthalmology, vol. 230, no. 6, pp. 511-517, 1992.

[32] Y. Kimura, M. Hangai, S. Morooka et al., "Retinal nerve fiber layer defects in highly myopic eyes with early glaucoma," Investigative Ophthalmology and Visual Science, vol. 53, no. 10, pp. 6472-6478, 2012.

[33] O. Arend, A. Remky, N. Plange, M. Kaup, and B. Schwartz, "Fluorescein leakage of the optic disc in glaucomatous optic neuropathy," Graefe's Archive for Clinical and Experimental Ophthalmology, vol. 243, no. 7, pp. 659-664, 2005.

[34] N. Plange, M. Kaup, A. Weber, A. Remky, and O. Arend, "Fluorescein filling defects and quantitative morphologic analysis of the optic nerve head in glaucoma," Archives of Ophthalmology, vol. 122, no. 2, pp. 195-201, 2004.

[35] N. Plange, M. Kaup, K. Huber, A. Remky, and O. Arend, "Fluorescein filling defects of the optic nerve head in normal tension glaucoma, primary open-angle glaucoma, ocular hypertension and healthy controls," Ophthalmic and Physiological Optics, vol. 26, no. 1, pp. 26-32, 2006.

[36] R. Sihota, R. Saxena, N. Taneja, P. Venkatesh, and A. Sinha, "Topography and fluorescein angiography of the optic nerve head in primary open-angle and chronic primary angle closure glaucoma," Optometry and Vision Science, vol. 83, no. 7, pp. 520 526, 2006.

[37] E. D. Talusan and B. Schwartz, "Fluorescein angiography. Demonstration of flow pattern of anterior ciliary arteries," Archives of Ophthalmology, vol. 99, no. 6, pp. 1074-1080, 1981.

[38] A. S. Hafez, R. L. Bizzarro, and M. R. Lesk, "Evaluation of optic nerve head and peripapillary retinal blood flow in glaucoma patients, ocular hypertensives, and normal subjects," American Journal of Ophthalmology, vol. 136, no. 6, pp. 1022-1031, 2003.

[39] N. Aizawa, Y. Yokoyama, N. Chiba et al., "Reproducibility of retinal circulation measurements obtained using laser speckle flowgraphy-NAVI in patients with glaucoma," Clinical Ophthalmology, vol. 5, no. 1, pp. 1171-1176, 2011.

[40] J.-C. Mwanza, J. D. Oakley, D. L. Budenz, R. T. Chang, O. J. Knight, and W. J. Feuer, "Macular ganglion cell-inner plexiform layer: automated detection and thickness reproducibility with spectral domain-optical coherence tomography in glaucoma," Investigative Ophthalmology \& Visual Science, vol. 52, no. 11, pp. 8323-8329, 2011.

[41] Y. Liang, J. C. Downs, B. Fortune, G. Cull, G. A. Cioffi, and L. Wang, "Impact of systemic blood pressure on the relationship between intraocular pressure and blood flow in the optic nerve head of nonhuman primates," Investigative Ophthalmology \& Visual Science, vol. 50, no. 5, pp. 2154-2160, 2009. 


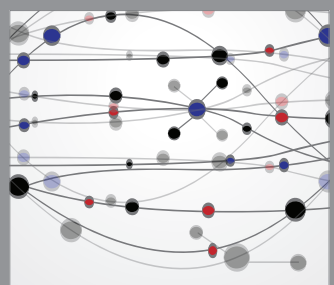

The Scientific World Journal
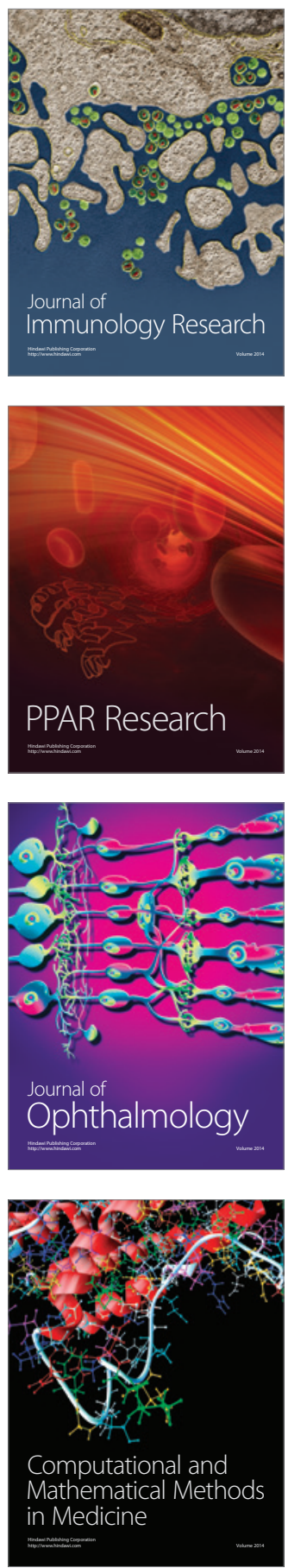

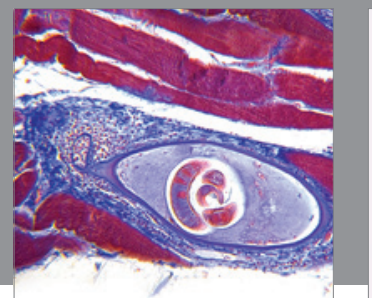

Gastroenterology

Research and Practice
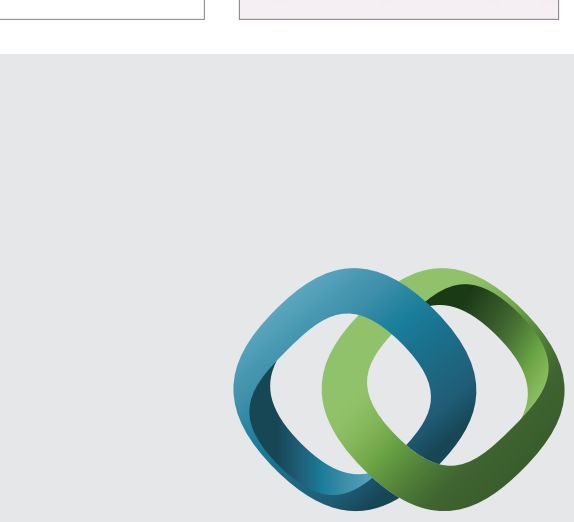

\section{Hindawi}

Submit your manuscripts at

http://www.hindawi.com
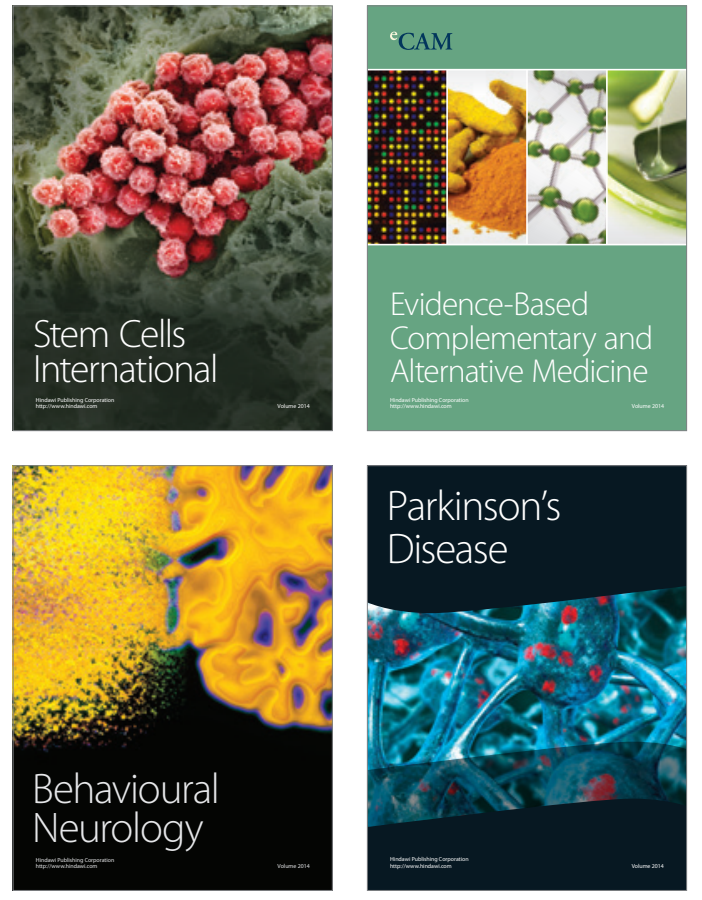
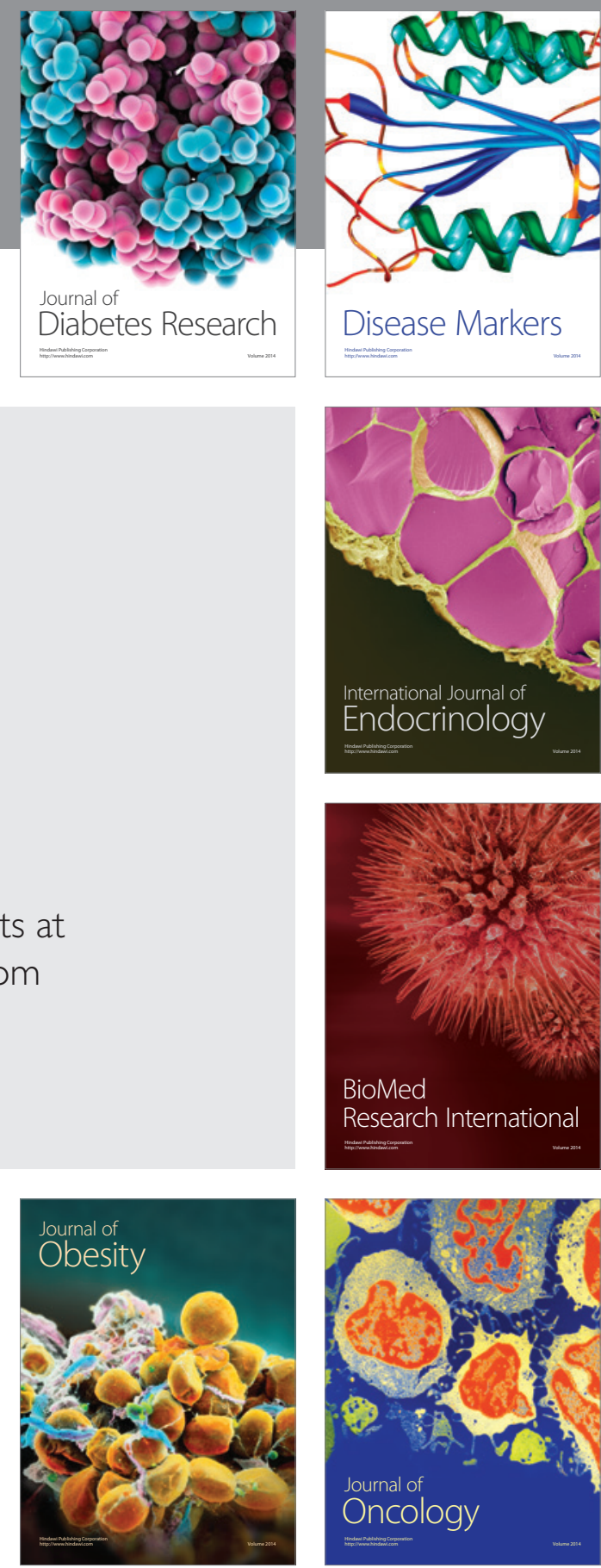

Disease Markers
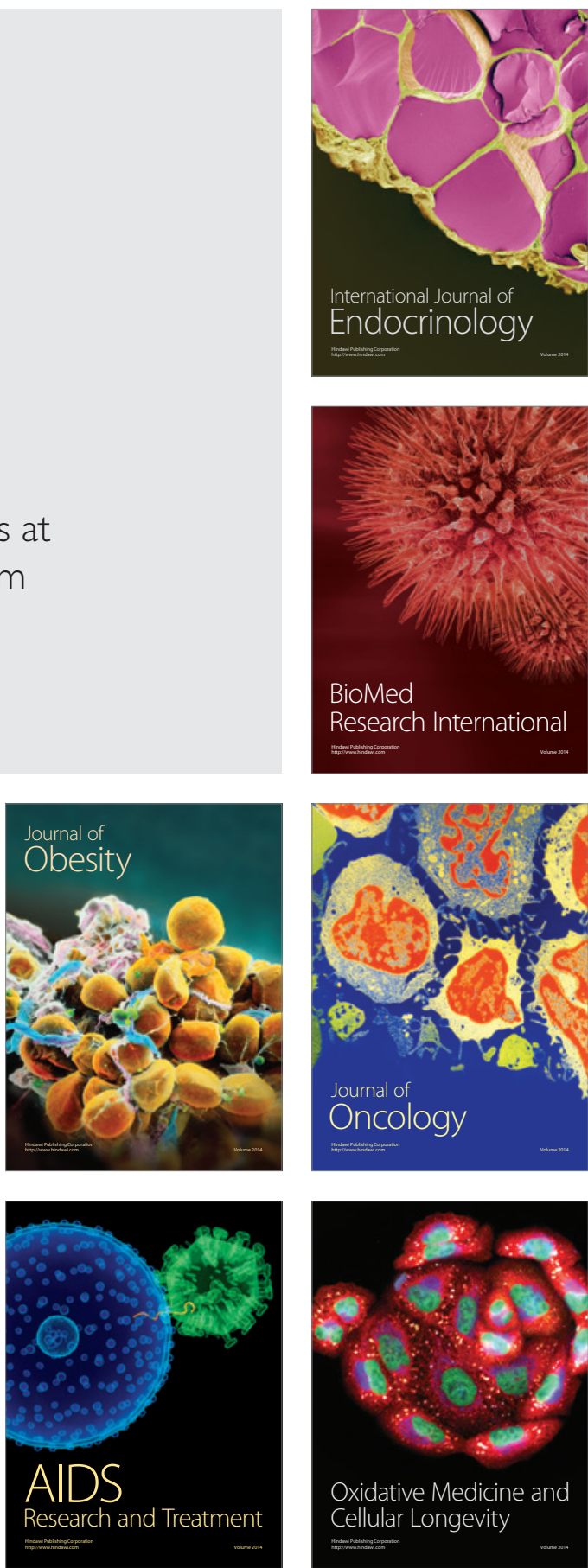